\title{
Almost Weakly Compact Operators
}

\author{
by \\ Ioana GHENCIU and Paul LEWIS \\ Presented by Gilles PISIER
}

Summary. Dunford-Pettis type properties are studied in individual Banach spaces as well as in spaces of operators. Bibasic sequences are used to characterize Banach spaces which fail to have the Dunford-Pettis property. The question of whether a space of operators has a Dunford-Pettis property when the dual of the domain and the codomain have the respective property is studied. The notion of an almost weakly compact operator plays a consistent and important role in this study.

1. Introduction. Throughout this paper $X, Y, E$, and $F$ will denote real Banach spaces. The unit ball of $X$ will be denoted by $B_{X}$ unless otherwise specified, and $X^{*}$ will denote the continuous linear dual of $X$. An operator $T: X \rightarrow Y$ will be a continuous and linear function. Of course, it is well known that $T: X \rightarrow Y$ is a compact (resp. weakly compact) operator if and only if $T^{*}\left(B_{Y^{*}}\right)$ is relatively compact (resp. relatively weakly compact) in $X^{*}$. The following equivalences (which one can easily derive directly from definitions) appear to be less well celebrated:

(a) $T: X \rightarrow Y$ is completely continuous iff $T^{*}\left(B_{Y^{*}}\right)$ is an $L$-subset of $X^{*}$.

(b) $T: X \rightarrow Y$ is unconditionally converging iff $T^{*}\left(B_{Y^{*}}\right)$ is a $V$-subset of $X^{*}$.

(c) $T: X \rightarrow Y$ is limited iff $T^{*}$ is $w^{*}$-norm sequentially continuous.

(d) $T\left(B_{X}\right)$ is a DP subset of $Y$ iff $T^{*}: Y^{*} \rightarrow X^{*}$ is completely continuous.

(e) $T\left(B_{X}\right)$ is a $V^{*}$-subset of $Y$ iff $T^{*}: Y^{*} \rightarrow X^{*}$ is unconditionally converging.

2000 Mathematics Subject Classification: 46B20, 46B25, 46B28.

Key words and phrases: Dunford-Pettis property, Dunford-Pettis sets, limited sets, $L$-sets, $w^{*}-w$ continuity, almost weakly compact operator. 
In this paper we will investigate some structural consequences of (a)-(e) as well as topological properties of distinguished sets naturally associated with the classes of operators mentioned above.

2. Definitions and notation. The set of all continuous linear transformations from $X$ to $Y$ will be denoted by $L(X, Y)$, and the compact operators will be denoted by $K(X, Y)$. The $w^{*}-w$ continuous (resp. $w^{*}-w$ continuous compact) maps from $X^{*}$ to $Y$ will be denoted by $L_{w^{*}}\left(X^{*}, Y\right)$ (resp. $\left.K_{w^{*}}\left(X^{*}, Y\right)\right)$.

The bounded subset $A$ of $X$ is called a Dunford-Pettis subset (resp. limited subset) of $X$ if each weakly null sequence in $X^{*}$ (resp. $w^{*}$-null sequence in $X^{*}$ ) tends to 0 uniformly on $A$. The bounded subset $A$ of $X^{*}$ is called an $L$-subset of $X^{*}$ if each weakly null sequence in $X$ tends to 0 uniformly on $A$, and the subset $S$ of $X$ is said to be weakly precompact provided that every bounded sequence from $S$ has a weakly Cauchy subsequence.

The operator $T: X \rightarrow Y$ is limited provided that $T\left(B_{X}\right)$ is a limited subset of $Y ; T$ is completely continuous (or Dunford-Pettis) if it maps weakly Cauchy sequences to norm convergent sequences; $T$ is unconditionally converging if it maps weakly unconditionally converging series to unconditionally converging series; and $T$ is almost weakly compact if $T\left(B_{X}\right)$ is weakly precompact. If every weakly compact operator defined on $X$ is completely continuous, then we say that $X$ has the Dunford-Pettis property (=DPP); see [9] and [1] for inventories of classical results related to the DPP. We denote the canonical unit vector basis of $c_{0}\left(\right.$ resp. $\left.\ell_{1}\right)$ by $\left(e_{n}\right)\left(\right.$ resp. $\left.\left(e_{n}^{*}\right)\right)$. The reader should consult Lindenstrauss and Tzafriri [29], Diestel [10], or [3] for undefined terminology and notation.

3. Almost weakly compact operators, limited sets, and Dunford-Pettis sets. Odell, Rosenthal, and Stegall [33, p. 377] showed that an operator $T: X \rightarrow Y$ is almost weakly compact if $L \circ T: X \rightarrow L_{1}$ is compact whenever $L: Y \rightarrow L_{1}$ is a completely continuous map. (The converse of this statement is straightforward.) In view of the connection between completely continuous operators and $L$-sets noted in (a) above, the Odell-Stegall-Rosenthal theorem appears to say that the $L$-subsets of $Y^{*}$ are sufficient to identify the almost weakly compact operators $T: X \rightarrow Y$. More precisely, we have the following.

TheOREM 3.1. An operator $T: X \rightarrow Y$ is almost weakly compact if and only if $T^{*}(A)$ is relatively compact in $X^{*}$ whenever $A$ is an L-subset of $Y^{*}$.

Proof. Suppose that $T: X \rightarrow Y$ is almost weakly compact, and let $A$ be an $L$-subset of $Y^{*}$. Let $B(A)$ be the Banach space (sup norm) of all bounded real-valued functions defined on $A$, and let $J: Y \rightarrow B(A)$ be the natural 
evaluation map, i.e., $J(y)(a)=a(y)$. Since $A$ is an $L$-set, $J$ is completely continuous. Then $J \circ T$ is compact, $A \subseteq J^{*}\left(B^{*}\right)\left(B^{*}=\right.$ unit ball of $\left.B(A)^{*}\right)$, and $T^{*} J^{*}\left(B^{*}\right)$ is relatively compact. Thus $T^{*}(A)$ is relatively compact.

Conversely, suppose that $T^{*}(A)$ is relatively compact for all $L$-subsets $A$ of $Y^{*}$. Let $L: Y \rightarrow L_{1}$ be a completely continuous operator. Then $L^{*}\left(B_{L_{\infty}}\right)$ is an $L$-subset of $Y^{*}$, and $T^{*}\left(L^{*}\left(B_{L_{\infty}}\right)\right)$ is relatively compact. Therefore $(L \circ T)^{*}$ and $L \circ T$ are compact. By the Odell-Rosenthal-Stegall theorem, $T$ is almost weakly compact.

COROLlary 3.2 ([14]). Every L-subset of $X^{*}$ is relatively compact iff $\ell_{1} \leftrightarrow X$.

Proof. Apply Rosenthal's $\ell_{1}$-theorem and the previous theorem to the identity operator $I: X \rightarrow X$.

It is well known that $\ell_{1} \hookrightarrow X$ if and only if $L_{1} \hookrightarrow X^{*}$ [10, p. 213], and that $L_{1}$ has the DPP [9]. Consequently, any normalized and weakly null sequence in $L_{1}$ is a non-relatively compact DP subset (therefore a nonrelatively compact $L$-subset) of $L_{1}$. Thus the preceding corollary can be restated.

Corollary 3.3 ([2, Cor. 7], [20, Thm. 1]). The following are equivalent:

(i) $\ell_{1} \nrightarrow X$.

(ii) Every L-subset of $X^{*}$ is relatively compact.

(iii) Every DP subset of $X^{*}$ is relatively compact.

(iv) Every weakly null DP sequence in $X^{*}$ is norm null.

(v) $L_{1} \nrightarrow X^{*}$.

(vi) The identity map from $X$ to $X$ is almost weakly compact.

The Odell-Rosenthal-Stegall theorem used completely continuous maps with range in $L_{1}$ to identify almost weakly compact operators. Weakly compact maps with range in $c_{0}$ can also be employed to identify such operators.

Theorem 3.4. If $T: Y \rightarrow X$ is an operator and $L \circ T: Y \rightarrow c_{0}$ is compact for all weakly compact operators $L: X \rightarrow c_{0}$, then $T$ is almost weakly compact.

Proof. Let $\left(y_{n}\right)$ be a sequence in $B_{Y}$. Suppose (by way of contradiction) that $\left(T\left(y_{n}\right)\right)$ has no weakly Cauchy subsequence. By Rosenthal's $\ell_{1}$-theorem, we can assume that $\left(T\left(y_{n}\right)\right) \sim\left(e_{n}^{*}\right)$. Let $S=\left[T\left(y_{n}\right)\right]$, an isomorph of $\ell_{1}$. Let $j: S \rightarrow c_{0}$ be the natural inclusion. Since $j$ naturally factors through $\ell_{2}$, $j$ is absolutely 2 -summing [32, Satz 2]. Now use the fact that all closed linear subspaces of an $L_{2}$-space are complemented and the constructions in Theorem 2 of [32] $\left(U^{0}=\left(B_{X^{*}}, w^{*}\right)\right)$ and on pp. 60-61 of [10] to obtain a weakly compact operator $J: X \rightarrow c_{0}$ which extends $j$. Then $J T: Y \rightarrow c_{0}$ 
is compact. But $J T\left(y_{n}\right)=j\left(e_{n}^{*}\right)=e_{n}$, and $\left(e_{n}\right)$ is certainly not relatively compact in $c_{0}$.

The weak precompactness of a DP set is well known and has been used extensively by many authors; e.g., see [1], [3], [13] and [20]. The usual proof of this result involves the Odell-Rosenthal-Stegall theorem. Theorem 3.4 yields a particularly quick argument for this fact.

Corollary 3.5. Every Dunford-Pettis subset of $X$ is weakly precompact.

Proof. If $K$ is a DP subset of $X, W$ is the closed and absolutely convex hull of $K$, and $T: \ell_{1}(W) \rightarrow X$ is defined by $T(f)=\sum_{w \in W} f(w) w$, then $T$ and $T^{*}$ are completely continuous, and $K \subseteq T\left(B_{\ell_{1}(W)}\right)$. If $L: X \rightarrow c_{0}$ is weakly compact, then a look at the adjoint immediately shows that $L \circ T$ is compact.

It is known that a set $A$ can be isometrically embedded in both $E$ and $F(F \subset E)$ and be a limited (Dunford-Pettis) subset of $E$ and fail to be a limited (Dunford-Pettis) subset of $F$. For example, Phillips' lemma shows that if $j: c_{0} \rightarrow \ell_{\infty}$ is the natural inclusion, then $j^{*}: \ell_{\infty}^{*} \rightarrow c_{0}^{*}$ is $w^{*}$-norm sequentially continuous; thus $A:=B_{c_{0}}$ is a limited set in $\ell_{\infty}$. Certainly $A$ fails to be a limited subset of $c_{0}$.

Also, if $B$ is any bounded subset of a separable reflexive space $R$ and $R$ is viewed as sitting in $C[0,1]$, then the fact that $C[0,1]$ has the DPP guarantees that $B$ is a Dunford-Pettis subset of $C[0,1]$. If $R$ is infinite-dimensional and $B=B_{R}$, then the identity map $I: R \rightarrow R$ clearly shows that $B$ is not a Dunford-Pettis subset of $R$. In fact, since the continuous linear image of a DP set is a DP set, $B$ is not a DP subset of any space in which $R$ is complemented.

We note that these observations, together with the easily established fact that the continuous linear image of a limited set is a limited set, lead to a particularly simple proof that $c_{0}$ is not complemented in $\ell_{\infty}$. For if $P: \ell_{\infty} \rightarrow c_{0}$ were a projection, then $P\left(B_{c_{0}}\right)=B_{c_{0}}$ would be a limited subset of $c_{0}$, and it is not.

The definitive result which determines when a basic sequence $\left(x_{n}\right)$ in $X$ is simultaneously equivalent to $\left(e_{n}\right)$ and limited in $X$ is due to Schlumprecht. See p. 36 of [35] for a proof.

THEOREM 3.6. If $\left(x_{n}\right)$ is a basic sequence in $X$ which is equivalent to $\left(e_{n}\right)$, then the following are equivalent:

(a) $\left(x_{n}\right)$ is a limited sequence in $X$.

(b) If $S$ is an infinite subset of $\mathbb{N}$, then $\overline{\operatorname{span}}\left\{x_{n}: n \in S\right\}$ is not complemented in $X$. 
The proof of Theorem 3.6 in [35] used Sobczyk's theorem: If $X$ is separable and $c_{0} \hookrightarrow X$, then $c_{0}$ is complemented in $X$. The proof of 3.6 immediately implies that the following is true.

If $B_{X^{*}}$ is $w^{*}$-sequentially compact, $\left(x_{n}\right)$ is a basic sequence in $X$ which is equivalent to $\left(e_{n}\right)$, and $\left(b_{n}\right)$ is any subsequence of $\left(x_{n}\right)$, then there is a subsequence $\left(c_{n}\right)$ of $\left(b_{n}\right)$ such that $\left[\left(c_{n}\right)\right]$ is complemented in $X$. We do not know if the full sequence $\left[\left(x_{n}\right)\right]$ is complemented in $X$. See Emmanuele [17] for a similar result.

The next result uses bibasic sequences to characterize when $\left(e_{n}\right)$ embeds as a limited sequence in $X$. In the process, we extend Theorem 4.5 of [3]. We refer the reader to [8], [3], and [36, p. 32] for a discussion of bibasic sequences $\left(x_{n}, f_{n}^{*}\right)$ in $X \times X^{*}$. In particular, note that if $\left(x_{n}, f_{n}^{*}\right)$ is a bibasic sequence in $X$ and $\left(x_{n}^{*}\right)$ is the a.s.c.f. for $\left(x_{n}\right)$, then $f_{k}^{*}$ is a continuous linear extension of $x_{k}^{*}$ to all of $X$ for each $k$. The problem of the equivalence of $\left(f_{n}^{*}\right)$ and $\left(x_{n}^{*}\right)$ as basic sequences has been studied by several authors; e.g., see [36].

It is known that $\ell_{\infty}$ contains limited sets which are not relatively weakly compact (see Example 1.1.8 of [35]). Further, Haydon [25] has given an example of a $C(K)$ space which is Grothendieck and does not contain $\ell_{\infty}$. Such a space must contain limited sets $S$ which are not relatively weakly compact [35, pp. 27-28]. We shall see that such limited sets generate bibasic sequences $\left(x_{n}, f_{n}^{*}\right)$ for which $\left(f_{n}^{*}\right) \nsim\left(x_{n}^{*}\right)$ in a very strong sense. In the next theorem, $t: \mathbb{N} \rightarrow \mathbb{N}$ is a strictly increasing function and $u_{t(n)}^{*}=\left.x_{t(n)}^{*}\right|_{\left[x_{t(j)}: j \in \mathbb{N}\right]}$.

THEOREM 3.7. If $S$ is a limited subset of $X$ which is not relatively weakly compact, then there is a a bibasic sequence $\left(x_{n}, f_{n}^{*}\right)$ in $S \times X^{*}$ so that if $t: \mathbb{N} \rightarrow \mathbb{N}$ and $u_{t(n)}^{*}$ are defined as above, then $\left(u_{t(n)}^{*}\right) \nsim\left(f_{t(n)}^{*}\right)$. Further $\left(e_{n}\right)$ embeds as a limited sequence in $X$ iff there is a bounded bibasic sequence $\left(x_{n}, f_{n}^{*}\right)$ in $X \times X^{*}$ so that $\left(x_{n}\right)$ is limited in $X$, unconditional, and $\left(f_{n}^{*}\right) \sim\left(x_{n}^{*}\right)$.

Proof. Let $\left(x_{n}\right)$ be a sequence in $S$ which has no weakly convergent subsequence. By Pełczyński's version of the Eberlein-Šmulian Theorem [10, p. 41], we may assume that $\left(x_{n}\right)$ is a seminormalized basic sequence. Let $\left(x_{n}^{*}\right)$ be the sequence of coefficient functionals, and let $\left(f_{n}^{*}\right)$ be a sequence of Hahn-Banach extensions to all of $X$. Suppose that $\left(f_{n}^{*}\right)$ has a $w^{*}$-Cauchy subsequence. Without loss of generality, assume that $f_{n}^{*}-f_{n+1}^{*} \stackrel{w^{*}}{\rightarrow} 0$. Then $\left\langle f_{n}^{*}-f_{n+1}^{*}, x_{n}\right\rangle \rightarrow 0$ since $\left(x_{n}\right)$ is limited. However, $\left\langle f_{n}^{*}-f_{n+1}^{*}, x_{n}\right\rangle=1$ for all $n$. Therefore $\left(f_{n}^{*}\right)$ has no $w^{*}$-Cauchy subsequence, and thus it has no weakly Cauchy subsequence. By Rosenthal's $\ell_{1}$-theorem, we may assume that $\left(f_{n}^{*}\right) \sim\left(e_{n}^{*}\right)$. The bibasic sequence $\left(x_{n}, f_{n}^{*}\right)$ satisfies the first conclusion of the theorem. For if $\left(f_{t(n)}^{*}\right)$ were equivalent to $\left(u_{t(n)}^{*}\right)$, then the proof of 
Lemma 2.2 in [28] would show that $\left(x_{t(n)}\right) \sim\left(e_{n}\right)$ and some subsequence of $\left(x_{n}\right)$ would be weakly null.

If there is an isomorphism $T: c_{0} \rightarrow X$ so that the sequence $\left(T\left(e_{n}\right)\right)$ is limited in $X$ and $\left(x_{n}^{*}\right)$ is the associated sequence of coefficient functionals, then $\left(x_{n}^{*}\right) \sim\left(e_{n}^{*}\right)$. If $\left(f_{n}^{*}\right)$ is any sequence of Hahn-Banach extensions of $\left(x_{n}^{*}\right)$ to all of $X$, then the strength of the $\ell_{1}$-norm ensures that $\left(f_{n}^{*}\right) \sim\left(x_{n}^{*}\right)$.

Conversely, suppose that the sequence $\left(x_{n}, f_{n}^{*}\right)$ satisfies the hypotheses of the concluding statement in the theorem. Let $L:\left[f_{n}^{*}\right] \rightarrow\left[x_{n}^{*}\right]$ be an isomorphism so that $L\left(f_{n}^{*}\right)=x_{n}^{*}$ for each $n$. Since $\left(x_{n}\right)$ is a limited sequence in $X$, some subsequence $\left(f_{n_{i}}^{*}\right)$ of $\left(f_{n}^{*}\right)$ is equivalent to $\left(e_{i}^{*}\right)$. Thus $\left(x_{n_{i}}^{*}\right) \sim\left(e_{i}^{*}\right)$. Consequently, we have an unconditional basic sequence $\left(x_{n}\right)$ and a subsequence $\left(x_{n_{i}}^{*}\right)$ of the coordinate functionals so that $\left(x_{n_{i}}^{*}\right) \sim\left(e_{i}^{*}\right)$ in $\left[\left(x_{n}\right)\right]^{*}$. Thus $\left(x_{n_{i}}\right) \sim\left(e_{i}\right)$ (see Lemma 1 in [28]).

Next we use bibasic sequences and the techniques in the proof of 1.3.1 in [35] to produce a strong characterization of spaces which do not have the DPP.

TheOREM 3.8. The Banach space $X$ fails to have the DPP iff there is a bounded bibasic sequence $\left(x_{n}, f_{n}^{*}\right) \in X \times X^{*}$ so that $\left(x_{n}\right),\left(x_{n}^{*}\right)$, and $\left(f_{n}^{*}\right)$ are weakly null.

Proof. Note first that $X$ has the DPP iff every weakly null sequence in $X$ is a Dunford-Pettis subset of $X$. Suppose then that $X$ fails to have the DPP, and let $\left(x_{n}\right)$ be a weakly null sequence in $X$ which is not Dunford-Pettis. By passing to a subsequence, we may (and do) assume that $\left(x_{n}\right)$ is a weakly null basic sequence (apply the Bessaga-Pełczyński selection principle [10]) which is not a Dunford-Pettis sequence in $X$ and none of its subsequences is a Dunford-Pettis sequence in $X$. Thus we may assume that there is a $w$-null sequence $\left(z_{n}^{*}\right)$ in $X^{*}$ and $z_{n}^{*}\left(x_{n}\right)=1$ for each $n$. Further, by passing to subsequences, we may assume that $\left(z_{n}^{*}\left(x_{m}\right)\right)_{m=1}^{\infty}$ converges for all $n$. Let $a_{n}=\lim _{m} z_{n}^{*}\left(x_{m}\right)$ for each $n$. By passing to another subsequence if necessary, one may assume that one of the following three cases holds:

(1) $a_{n}=0$ for each $n$.

(2) $a_{n} \neq 0$ for each $n$, and $a_{n} \rightarrow 0$.

(3) There is an $\varepsilon>0$ for which $\left|a_{n}\right|>\varepsilon, n \in \mathbb{N}$.

In (1) we set $y_{n}^{*}=z_{n}^{*}$ for each $n$. Now suppose that (2) holds. By passing to subsequences, we assume that $z_{n-1}^{*}\left(x_{n}\right)<1 / 4$ for each $n$. Define $r_{n}$ to be $\left\langle z_{n}^{*}-\left(a_{n} / a_{n-1}\right) z_{n-1}^{*}, x_{n}\right\rangle$ for $n \in \mathbb{N}$. Since $a_{n} / a_{n-1} \rightarrow 1$, we may assume that $r_{n} \geq 1 / 2$ for each $n$. Set $y_{n}^{*}=\left(1 / r_{n}\right)\left(z_{n}^{*}-\left(a_{n} / a_{n-1}\right) z_{n-1}^{*}\right)$, and note that $y_{n}^{*} \stackrel{w}{\rightarrow} 0,\left\langle y_{n}^{*}, x_{n}\right\rangle=1$, and $\lim _{m} y_{n}^{*}\left(x_{m}\right)=0$ for each $n$.

Suppose next that (3) holds. In this case, the sequence $\left(a_{n} / a_{n+1}\right)$ is bounded. Set $r_{n}=\left\langle z_{n}^{*}-\left(a_{n} / a_{n+1}\right) z_{n+1}^{*}, x_{n}\right\rangle$. Since $\left(z_{n}^{*}\right)$ is weakly null, we 
may assume (as above) that $r_{n} \geq 1 / 2$ for each $n$. Set $y_{n}^{*}=\left(1 / r_{n}\right)\left(z_{n}^{*}-\right.$ $\left.\left(a_{n} / a_{n+1}\right) z_{n+1}^{*}\right)$. Then, as in the preceding paragraph, $y_{n}^{*}\left(x_{n}\right)=1, y_{n}^{*} \stackrel{w}{\rightarrow} 0$, and $y_{n}^{*}\left(x_{m}\right) \stackrel{m}{\rightarrow} 0$ for each $n$.

The reader should note that in all three cases one has $\lim _{m} y_{m}^{*}\left(x_{n}\right)=$ $\lim _{m} y_{n}^{*}\left(x_{m}\right)=0$ for each $n$. By passing to further subsequences if necessary, we assume that $\sum_{m, m \neq n}\left|y_{n}^{*}\left(x_{m}\right)\right|<1 / 2, n \in \mathbb{N}$.

Define $T: X \rightarrow c_{0}$ by $T(x)=\left(y_{n}^{*}(x)\right)$. It is not difficult to check that $\left(T\left(x_{n}\right)\right) \sim\left(e_{n}\right)$. Further, $T^{*}\left(e_{n}^{*}\right)=y_{n}^{*}$ for each $n$. Thus $T^{*}$ and $T$ are weakly compact operators. Let $Y_{0}=\overline{\operatorname{span}}\left\{T\left(x_{n}\right): n \in \mathbb{N}\right\}=\left[\left(T\left(x_{n}\right)\right)\right]$. Define $S_{0}: Y_{0} \rightarrow c_{0}$ by $S_{0}\left(T\left(x_{n}\right)\right)=e_{n}$ for each $n$. Now $Y_{0}$ is (isomorphic to) a separable subspace of $c_{0}$, and $c_{0}$ is separably injective. Let $S: c_{0} \rightarrow c_{0}$ be a continuous linear extension of $S_{0}$, and consider the operator $S T: X \rightarrow c_{0}$. Let $f_{n}^{*}=(S T)^{*}\left(e_{n}^{*}\right)$ for each $n$. Since $S T$ is weakly compact and $e_{n}^{*} \stackrel{w^{*}}{\rightarrow} 0$, it follows that $f_{n}^{*} \stackrel{w}{\rightarrow} 0$; i.e., the adjoint of a weakly compact operator is $w^{*}-w$ continuous. Further, $f_{n}^{*}\left(x_{m}\right)=\left\langle e_{n}^{*}, e_{m}\right\rangle=\delta_{n m}$. Another application of the Bessaga-Pełczyński selection principle allows us to assume that $\left(f_{n}^{*}\right)$ is a weakly null basic sequence in $X^{*}$. Let $x_{n}^{*}=\left.f_{n}^{*}\right|_{\left[\left(x_{n}\right)\right]}$. To see that $\left(x_{n}^{*}\right)$ is weakly null, notice that the restriction operator $R: X^{*} \rightarrow\left[\left(x_{n}\right)\right]^{*}$ maps weakly null sequences to weakly null sequences. This completes the proof of one implication in the theorem, and the converse implication follows from the definition.

REMARK. By localizing the preceding argument, one sees that if $K$ is a bounded subset which is not a Dunford-Pettis subset of $X$, then one can find a sequence $\left(x_{n}\right)$ in $K$ and a weakly null sequence $\left(x_{n}^{*}\right)$ in $X^{*}$ so that $x_{n}^{*}\left(x_{m}\right)=\delta_{n m}$. Lemma 1.3.1 of [35] showed that if a bounded subset $K$ of $X$ is not limited in $X$, then there exists an operator $T: X \rightarrow c_{0}$ so that $\left\{e_{n}\right.$ : $n \in \mathbb{N}\} \subseteq T(K)$. The proof of 3.8 shows that $K$ is not a DP subset of $X$ iff there is a weakly compact operator $T: X \rightarrow c_{0}$ so that $\left\{e_{n}: n \in \mathbb{N}\right\} \subseteq T(K)$.

If $\left(x_{n}\right)$ is a basic sequence in $X$ and $\left(x_{n}\right) \sim\left(e_{n}\right)$, then $\left(x_{n}\right)$ is always a DP sequence in $X$, and Theorems 3.6 and 3.7 investigate when $\left(x_{n}\right)$ is a limited sequence in $X$. If $\left(x_{n}\right) \sim\left(e_{n}^{*}\right)$, then $\left(x_{n}\right)$ is never a limited or a DP sequence in $X$. However, $\left(e_{n}^{*}\right)$ may embed as a $V^{*}$-subset of $X$. Also, $\left(e_{n}^{*}\right)$ may embed as a $V$-subset of $X^{*}$.

A bounded subset $A$ of $X$ (resp. $A$ of $X^{*}$ ) is called a $V^{*}$-subset of $X$ (resp. a $V$-subset of $X^{*}$ ) provided that

$$
\begin{gathered}
\lim _{n}\left(\sup \left\{\left|x_{n}^{*}(x)\right|: x \in A\right\}\right)=0 \\
\left(\text { resp. } \lim _{n}\left(\sup \left\{\left|x^{*}\left(x_{n}\right)\right|: x^{*} \in A\right\}\right)=0\right)
\end{gathered}
$$

for each $w u c$ series $\sum x_{n}^{*}$ in $X^{*}$ (resp. wuc series $\sum x_{n}$ in $X$ ). A Banach space $X$ has property $V$ if every $V$-subset of $X^{*}$ is relatively weakly com- 
pact, and $X$ has property $V^{*}$ if every $V^{*}$-subset of $X$ is relatively weakly compact [31].

Emmanuele [15] and Bombal [4] determined when $\left(e_{n}^{*}\right)$ embeds as a $V^{*}-$ sequence in $X$.

TheOREM 3.9. If $\left(x_{n}\right)$ is a sequence in $X$ and $\left(x_{n}\right) \sim\left(e_{n}^{*}\right)$, then the following are equivalent:

(1) $\left\{x_{n}: n \geq 1\right\}$ is a $V^{*}$-set in $X$.

(2) If $N$ is an infinite subset of $\mathbb{N}$, then $\left[x_{n}: n \in N\right]$ is not complemented in $X$.

Since every limited subset of $X$ is a DP subset of $X$, it is clear that every limited subset of $X$ is weakly precompact. Theorem 4.2 of [3] points out that the class of bounded and weakly precompact sets is properly contained in the class of $V^{*}$-sets. Since every bounded subset of $\ell_{\infty}$ is a $V$-subset of $\ell_{\infty}$, it is immediate that any isomorphic copy of $\left(e_{n}^{*}\right)$ contained in $\ell_{\infty}$ is a $V$-subset of $\ell_{\infty}$ which is not weakly precompact. Further, a direct interpretation of Theorem 3.10 in terms of $V$-sets fails: one can have $\left(x_{n}^{*}\right) \sim\left(e_{n}^{*}\right),\left(x_{n}^{*}\right)$ is a $V$-sequence in $X^{*}$, and $\left[x_{n}^{*}: n \in A\right]$ is complemented in $X^{*}$ for all non-empty $A \subseteq \mathbb{N}$.

Pełczyński gave an operator-theoretic characterization of spaces $X$ which have property $V$ : Every unconditionally converging operator with domain $X$ is weakly compact iff every $V$-subset of $X^{*}$ is relatively weakly compact. In the next theorem, we give elementary operator-theoretic characterizations of weak precompactness, relative weak compactness, and relative norm compactness for both $V$-sets and $V^{*}$-sets. The argument contains the theorem in [31] just cited.

THEOREM 3.10 .

(1) The following are equivalent:

(1)(i) If $T: Y \rightarrow X^{*}$ is an operator and $\left.T^{*}\right|_{X}$ is unconditionally converging, then $T$ is almost weakly compact (weakly compact, resp. compact).

(1)(ii) Same as (1)(i) with $Y=\ell_{1}$.

(1)(iii) Every $V$-subset of $X^{*}$ is weakly precompact (relatively weakly compact, resp. relatively compact).

(2) The following are equivalent:

(2)(i) If $T: Y \rightarrow X$ is an operator so that $T^{*}: X^{*} \rightarrow Y^{*}$ is unconditionally converging, then $T$ is almost weakly compact (weakly compact, resp. compact).

(2)(ii) Same as (2)(i) with $Y=\ell_{1}$. 
(2)(iii) Every $V^{*}$-subset of $X$ is weakly precompact (relatively weakly compact, resp. relatively compact).

Proof. We will show that (1)(i) $\Rightarrow(1)($ ii $) \Rightarrow(1)($ iii $) \Rightarrow(1)($ i) in the weakly precompact case, as well as $(2)(\mathrm{i}) \Rightarrow(2)(\mathrm{ii}) \Rightarrow(2)(\mathrm{iii}) \Rightarrow(2)(\mathrm{i})$ in the compact case. These two arguments are similar, and the arguments for all the remaining implications in the theorem follow the same pattern.

(1) (weakly precompact) Certainly (i) implies (ii). Now suppose that $A$ is a $V$-subset of $X^{*}$, and let $\left(x_{n}^{*}\right)$ be a sequence in $A$. Define $T: \ell_{1} \rightarrow X^{*}$ by $T(b)=\sum b_{i} x_{i}^{*}$. Since the closed absolutely convex hull of $\left(x_{i}^{*}\right)$ is a $V$-subset of $X^{*},\left.T^{*}\right|_{X}$ is unconditionally converging. Thus $T$ is almost weakly compact, and $\left(T\left(e_{n}^{*}\right)\right)=\left(x_{n}^{*}\right)$ has a weakly Cauchy subsequence. To see that (iii) $\Rightarrow(\mathrm{i})$, note that $T\left(B_{Y}\right)$ is a $V$-subset of $X^{*}$ iff $\left.T^{*}\right|_{X}$ is unconditionally converging.

(2) (compact) Certainly (i) implies (ii). Suppose that $A$ is a $V^{*}$-subset of $X$, and let $\left(x_{n}\right)$ be a sequence in $A$. Define $T: \ell_{1} \rightarrow X$ as above, and note that $T^{*}$ is unconditionally converging. Thus $T$ is compact, and $\left(x_{n}\right)$ has a norm converging subsequence. To see that (iii) $\Rightarrow(\mathrm{i})$, use (e) in the introduction.

Theorem 3.10 facilitates a particularly quick proof of Theorems 1 and $1^{\prime}$ of [31].

Corollary 3.11. If $H$ is a compact Hausdorff space and $X$ is reflexive, then $C(H, X)$ has property $V$.

Proof. If $T: Y \rightarrow C(H, X)^{*}$ is an operator and $L=\left.T^{*}\right|_{C(H, X)}$ is unconditionally converging, then $L$ is strongly bounded [7], [37], [12]. Since $X$ is reflexive, $L$ is weakly compact ([7, Thm. 4.1]). Therefore $T$ is weakly compact, and the desired conclusion follows from part (1) of the preceding theorem.

Observations (c) and (d) in the introduction, the fact that the closed absolutely convex hull of a DP (resp. limited) subset of $X$ is a DP (resp. limited) subset of $X$, and the arguments in 3.10 yield the next result.

THEOREM 3.12 .

(1) The following are equivalent:

(1)(i) If $T: Y \rightarrow X$ is an operator and $T^{*}: X^{*} \rightarrow Y^{*}$ is completely continuous, then $T$ is weakly compact (resp. compact).

(1)(ii) Same as (1)(i) with $Y=\ell_{1}$.

(1)(iii) Every DP subset of $X$ is relatively weakly compact (resp. relatively compact).

(2) The following are equivalent: 
(2)(i) If $T: Y \rightarrow X$ is an operator and $T^{*}: X^{*} \rightarrow Y^{*}$ is $w^{*}$-norm sequentially continuous, then $T$ is weakly compact (resp. compact).

(2)(ii) Same as (2)(i) with $Y=\ell_{1}$.

(2)(iii) Every limited subset of $X$ is relatively weakly compact (resp. relatively compact).

Corollary 3.13 ([6, parts 5 and 6$])$. If $X$ is reflexive or separable, then every limited subset of $X$ is relatively compact.

Proof. In either case, a $w^{*}$-norm sequentially continuous adjoint $T^{*}$ : $X^{*} \rightarrow Y^{*}$ must be compact.

Corollary 3.14. If $X^{*}$ is isomorphic to a $C(K)$-space, then every DP subset of $X$ is relatively weakly compact.

Proof. If $X^{*}$ is a $C(K)$-space and $T^{*}: X^{*} \rightarrow Y^{*}$ is completely continuous, then $T^{*}$ is unconditionally converging and thus weakly compact [31].

4. Spaces of operators. This section will be primarily concerned with studying the following two (related) questions:

(A) Does a space of operators have a certain Dunford-Pettis type property when the codomain and the dual of the domain have this property?

(B) Does $c_{0}$ embed isomorphically into a space of operators?

If $X$ is infinite-dimensional and $c_{0} \hookrightarrow L(X, Y)$, then $\ell_{\infty} \hookrightarrow L(X, Y)$ [27], and thus $L(X, Y)$ must contain a limited copy of $\left(e_{n}\right)$ as well as limited sets which are not relatively weakly compact. In 1.3.6 of [35], Schlumprecht showed that if $X$ contains a copy of $c_{0}$-whether the corresponding copy of $\left(e_{n}\right)$ is limited or not - then $\left(e_{n}\right)$ does embed as a non-limited sequence in the least crossnorm tensor product completion $X \otimes_{\lambda} Y$ of $X$ and $Y$. We say that $X$ has (DPrcP) if every DP subset of $X$ is relatively compact [20]; $X$ has $\mathrm{RDP}^{*}$ (or $X \in\left(\mathrm{RDP}^{*}\right)$ ) if every DP subset of $X$ is relatively weakly compact [3]; $X$ is a Gelfand-Phillips space if every limited subset of $X$ is relatively compact, and $X$ has the (BD) property if every limited subset of $X$ is relatively weakly compact. If $\ell_{1} \hookrightarrow X$, then $X$ has the (BD) property [6]. $X$ has RDP* whenever $X$ has $V^{*}$ or $X$ is weakly sequentially complete [3]. Also, $X^{*}$ has $\mathrm{RDP}^{*}$ whenever $X$ has $V$. If $c_{0} \hookrightarrow L(X, Y)$, then $L(X, Y)$ fails to belong to any of the classes of spaces just defined.

One can find numerous references to papers which study the embedability of $c_{0}$ in spaces of operators in [19], [18], and [27]. Specifically we note that [19], [18], and [27] point out that if the Banach space $X$ has an unconditional finite-dimensional decomposition, then $c_{0} \hookrightarrow K(X, X)$. However, is $c_{0}$ com- 
plemented in these spaces? Emmanuele gave a positive answer in Theorem 18 of [21].

THEOREM 4.1. Let $c_{0}$ embed in $K_{w^{*}}\left(X^{*}, Y\right)$. If $Y$ is a Gelfand-Phillips space, then $c_{0}$ embeds complementably into $K_{w^{*}}\left(X^{*}, Y\right)$.

The techniques of Schlumprecht [35, Corollary 1.3.6] which were used to prove Theorem 4.1 can also be employed with different hypotheses to produce complemented copies of $c_{0}$ in $K_{w^{*}}\left(X^{*}, Y\right)$.

Theorem 4.2. Suppose that $X$ and $Y$ are infinite-dimensional Banach spaces.

(i) If $c_{0} \hookrightarrow X$ or $c_{0} \hookrightarrow Y$, then $K_{w^{*}}\left(X^{*}, Y\right)$ contains a copy of $c_{0}$ which is complemented in $K_{w^{*}}\left(X^{*}, Y\right)$.

(ii) If $c_{0} \longleftrightarrow X, B_{Y^{*}}$ is $w^{*}$-sequentially compact, and $c_{0} \hookrightarrow K_{w^{*}}\left(X^{*}, Y\right)$, then $c_{0}$ is complemented in $K_{w^{*}}\left(X^{*}, Y\right)$.

For completeness, we give a proof of Theorem 4.2.

Proof. (i) Let $\left(x_{n}\right)$ be a sequence of normalized vectors in $X$ so that $\left(x_{n}\right) \sim\left(e_{n}\right)$. Let $\left(x_{n}^{*}\right)$ be a sequence of norm one functionals in $X^{*}$ so that $x_{n}^{*}\left(x_{n}\right)=\left\|x_{n}\right\|$ for each $n$, and suppose that $\left(y_{n}^{*}\right)$ is a $w^{*}$-null sequence of norm one in $Y^{*}$. For each $n \in \mathbb{N}$, choose $y_{n} \in Y$ so that $1 \leq\left\|y_{n}\right\| \leq 2$ and $y_{n}^{*}\left(y_{n}\right)=1$. Let $T \in K_{w^{*}}\left(X^{*}, Y\right)$, and observe that $T^{*}$ is also $w^{*}-w$ continuous and compact. Therefore $\left\|T^{*}\left(y_{n}^{*}\right)\right\| \rightarrow 0$. Consequently, if one defines $x_{n}^{*} \otimes y_{n}^{*}(T)$ to be $y_{n}^{*}\left(T\left(x_{n}^{*}\right)\right)=\left\langle T^{*}\left(y_{n}^{*}\right), x_{n}^{*}\right\rangle$, then $\left(x_{n}^{*} \otimes y_{n}^{*}\right)$ is a $w^{*}$-null sequence in the dual of $K_{w^{*}}\left(X^{*}, Y\right)$. Moreover, $\left(x_{n} \otimes y_{n}\right)$ has a natural interpretation as a sequence in $K_{w^{*}}\left(X^{*}, Y\right)$ [11], and then the argument in 1.3.6 of [35] shows that $\left(x_{n} \otimes y_{n}\right) \sim\left(e_{n}\right)$. Now $\left\langle x_{n}^{*} \otimes y_{n}^{*}, x_{n} \otimes y_{n}\right\rangle=x_{n}^{*}\left(x_{n}\right) y_{n}^{*}\left(y_{n}\right)=$ $\left\|x_{n}\right\| \nrightarrow 0$, and $\left(x_{n} \otimes y_{n}\right)$ is not limited in $K_{w^{*}}\left(X^{*}, Y\right)$. Note that we obtain the same construction if we assume that $c_{0} \hookrightarrow Y$. An application of Theorem 3.6 gives that $\left[\left(x_{n} \otimes y_{n}\right)\right]$ is complemented in $K_{w^{*}}\left(X^{*}, Y\right)$.

(ii) Suppose that $\left(T_{n}\right) \sim\left(e_{n}\right)$ in $K_{w^{*}}\left(X^{*}, Y\right)$. Then $\sum T_{n}$ is weakly unconditionally convergent. Without loss of generality, suppose that $\left\|T_{n}\right\|=1$ for each $n$, and let $\left(x_{n}^{*}\right)$ be a sequence in $B_{X^{*}}$ so that $\left\|T_{n}\left(x_{n}^{*}\right)\right\|>1 / 2$. Now let $\left(y_{n}^{*}\right)$ be a sequence in $B_{Y^{*}}$ so that

$$
\left\langle y_{n}^{*}, T_{n}\left(x_{n}^{*}\right)\right\rangle=\left\|T_{n}\left(x_{n}^{*}\right)\right\|>1 / 2 .
$$

Further, since $B_{Y^{*}}$ is $w^{*}$-sequentially compact, we may assume that $y_{n}^{*} \stackrel{w^{*}}{\rightarrow} y^{*}$. Now use the fact that $\sum T_{n}$ is weakly unconditionally convergent to see that $\sum T_{n}^{*}\left(y^{*}\right)$ is weakly unconditionally convergent-and thus unconditionally convergent -in $X$. Therefore $\left\langle y^{*}, T_{n}\left(x_{n}^{*}\right)\right\rangle \leq\left\|T_{n}^{*}\left(y^{*}\right)\right\| \rightarrow 0$. Consequently,

$$
\left\langle y_{n}^{*}-y^{*}, T_{n}\left(x_{n}^{*}\right)\right\rangle=\left\langle y_{n}^{*}, T_{n}\left(x_{n}^{*}\right)\right\rangle-\left\langle y^{*}, T_{n}\left(x_{n}^{*}\right)\right\rangle \geq 1 / 2-1 / 4
$$


for large $n$. Specifically,

$$
\left\langle T_{n}, x_{n}^{*} \otimes\left(y_{n}^{*}-y^{*}\right)\right\rangle \not \rightarrow 0 .
$$

Since $\left(x_{n}^{*} \otimes\left(y_{n}^{*}-y^{*}\right)\right)$ is $w^{*}$-null in the dual of $K_{w^{*}}\left(X^{*}, Y\right),\left(T_{n}\right)$ is not limited in this space of operators. Apply Theorem 3.6.

REMARK. Theorems 3.7, 4.2, and 3.6 (Schlumprecht's result) make it clear that a Banach space $X$ may contain a copy of $\left(e_{n}\right)$ which is limited in $X$ as well as a copy which fails to be limited in $X$. In particular, if there are copies of $\left(e_{n}\right)$ in $X$ (or $Y$ ) which are limited in $X$ (or $Y$ ), then the natural isometric copies of these spaces in $K_{w^{*}}\left(X^{*}, Y\right)$ will produce copies of $\left(e_{n}\right)$ which are limited in this space of operators. The complemented copy of $\left(e_{n}\right)$ is not limited in $K_{w^{*}}\left(X^{*}, Y\right)$. Since $K(X, Y) \cong K_{w^{*}}\left(X^{* *}, Y\right)$, notice also that if $c_{0} \hookrightarrow X^{*}, B_{Y^{*}}$ is $w^{*}$-sequentially compact, and $c_{0} \hookrightarrow K(X, Y)$, then $c_{0} \stackrel{c}{\hookrightarrow} K(X, Y)$. Consequently, $c_{0} \stackrel{c}{\hookrightarrow} K\left(\ell_{2}, \ell_{2}\right)$, and thus $\left(e_{n}\right)$ embeds as a non-limited sequence in $K\left(\ell_{2}, \ell_{2}\right)$.

Recall that $X$ has the Schur property precisely when weak and norm convergence of sequences in $X$ coincide. Lust [30] and Ryan [34] showed that $L_{w^{*}}\left(X^{*}, Y\right)$ (resp. $L(X, Y)$ ) has the Schur property if and only if $X$ and $Y$ (resp. $X^{*}$ and $Y$ ) have the Schur property. Thus $L\left(c_{0}, \ell_{1}\right)$ is a Schur space, and $c_{0} \nrightarrow L\left(c_{0}, \ell_{1}\right)$. Note also that $L\left(c_{0}, \ell_{1}\right)=K\left(c_{0}, \ell_{1}\right)$. This equality is common to all spaces $L(X, Y)$ which have the Schur property. We give a brief argument for completeness.

Theorem 4.3. If $L(X, Y)$ is a Schur space, then $L(X, Y)=K(X, Y)$.

Proof. Suppose that $T \in L(X, Y)$ and $T$ is not compact. Since $Y$ has the Schur property, $T$ cannot be almost weakly compact. Thus there must be a sequence in $B_{X}$ without a weakly Cauchy subsequence, and Rosenthal's $\ell_{1}$-theorem guarantees that $\ell_{1} \hookrightarrow X$. Therefore $L_{1} \hookrightarrow X^{*}$ [10, p. 212], and $X^{*}$ cannot be a Schur space.

We remark that the proof of Theorem 4.3 could have been given using the fact that $X^{*}$ has the Schur property iff $X$ has the DPP and $\ell_{1} \hookrightarrow X$.

In [23] we proved the following theorem.

TheOREM 4.4. If $L_{w^{*}}\left(E^{*}, F\right)=K_{w^{*}}\left(E^{*}, F\right)$ and $E$ and $F$ are weakly sequentially complete, then $K_{w^{*}}\left(E^{*}, F\right)$ is weakly sequentially complete.

As a consequence, we deduce that $K_{w^{*}}\left(E^{*}, F\right)$ has $\mathrm{RDP}^{*}$ if $L_{w^{*}}\left(E^{*}, F\right)=$ $K_{w^{*}}\left(E^{*}, F\right)$ and both $E$ and $F$ are weakly sequentially complete.

Next we investigate sufficient conditions for a subset of $K_{w^{*}}\left(X^{*}, Y\right)$ to be relatively weakly compact. If $H \subseteq L_{w^{*}}\left(X^{*}, Y\right), x^{*} \in X^{*}$ and $y^{*} \in Y^{*}$, let $H\left(x^{*}\right)=\left\{T\left(x^{*}\right): T \in H\right\}$ and $H^{*}\left(y^{*}\right)=\left\{T^{*}\left(y^{*}\right): T \in H\right\}$. We begin with three lemmas which are similar to results in [26]. In Corollary 2 of [26], Kalton used these results to show that if $X$ and $Y$ are reflexive 
and $L(X, Y)=K(X, Y)$, then $K(X, Y)$ is reflexive. Kalton's lemmas are extended to the $w^{*}-w$ operator case. Theorem 4.8 below extends Corollary 2 of $[26]$.

Let $U$ denote the unit ball of $X^{*}$ with the $w^{*}$ topology and $V$ denote the unit ball of $Y^{*}$ with the $w^{*}$ topology. For $T$ in $L_{w^{*}}\left(X^{*}, Y\right)$, define $\chi_{T}$ : $U \times V \rightarrow \mathbb{R}$ by $\chi_{T}\left(x^{*}, y^{*}\right)=y^{*}\left(T x^{*}\right)$.

LEMMA 4.5. The mapping $T \mapsto \chi_{T}$ defines a linear isometry of the space $K_{w^{*}}\left(X^{*}, Y\right)$ onto a closed subspace of $C(U \times V)$.

Proof. Suppose $x_{\alpha}^{*} \stackrel{w^{*}}{\rightarrow} x^{*} \in U$ and $y_{\alpha}^{*} \stackrel{w^{*}}{\rightarrow} y^{*} \in V$. We have

$$
\begin{aligned}
\left|\chi_{T}\left(x_{\alpha}^{*}, y_{\alpha}^{*}\right)-\chi_{T}\left(x^{*}, y^{*}\right)\right| & =\left|y_{\alpha}^{*}\left(T x_{\alpha}^{*}\right)-y^{*}\left(T x^{*}\right)\right| \\
& \leq\left|y_{\alpha}^{*}\left(T x_{\alpha}^{*}-T x^{*}\right)\right|+\left|\left(y_{\alpha}^{*}-y^{*}\right)\left(T x^{*}\right)\right| \\
& \leq|| T x_{\alpha}^{*}-T x^{*} \|+\left|\left(y_{\alpha}^{*}-y^{*}\right)\left(T x^{*}\right)\right| .
\end{aligned}
$$

Since $T$ is $w^{*}-w$ continuous and compact, $T$ is $w^{*}$-norm continuous, and thus $\left\|T x_{\alpha}^{*}-T x^{*}\right\| \rightarrow 0$. Also, $\left|\left(y_{\alpha}^{*}-y^{*}\right)\left(T x^{*}\right)\right| \rightarrow 0$ because $y_{\alpha}^{*} \stackrel{w^{*}}{\rightarrow} y^{*}$. Thus $\chi_{T}\left(x_{\alpha}^{*}, y_{\alpha}^{*}\right) \rightarrow \chi_{T}\left(x^{*}, y^{*}\right)$ and $\chi_{T} \in C(U \times V)$. Since $\left\|\chi_{T}\right\|=\|T\|$ and $T \mapsto \chi_{T}$ is linear, the conclusion follows.

Let wot denote the weak operator topology on $L(X, Y): T_{n} \rightarrow T$ (wot) provided that $\left\langle T_{n}(x), y^{*}\right\rangle \rightarrow\left\langle T(x), y^{*}\right\rangle$ for all $x \in X$ and $y^{*} \in Y^{*}$. Following [26], we let $w^{\prime}$ denote the dual weak operator topology defined by the functionals $T \mapsto x^{* *} T^{*}\left(y^{*}\right), x^{* *} \in X^{* *}, y^{*} \in Y^{*}$.

Lemma 4.6. Let $A$ be a subset of $K_{w^{*}}\left(X^{*}, Y\right)$. Then $A$ is weakly compact if and only if $A$ is wot-compact.

Proof. Suppose $A$ is wot-compact and let $\chi(A)$ be $\left\{\chi_{T}: T \in A\right\}$. Let $\left(T_{\alpha}\right)$ be a net in $A$ and $\left(T_{\beta}\right)$ be a wot-convergent subnet. If $T_{\beta} \rightarrow T$ (wot), then $\chi_{T_{\beta}}\left(x^{*}, y^{*}\right) \rightarrow \chi_{T}\left(x^{*}, y^{*}\right)$ for all $x^{*} \in X^{*}$ and $y^{*} \in Y^{*}$, and $\chi(A)$ is compact in the topology of pointwise convergence on $C(U \times V)$. By Grothendieck's result [24], $\chi(A)$ is weakly compact in $C(U \times V)$, and $A$ is weakly compact by the preceding result.

The other implication is clear since wot is weaker than the weak topology on $K_{w^{*}}\left(X^{*}, Y\right)$.

LEMMA 4.7. Let $\left(T_{n}\right)$ be a sequence of $w^{*}-w$ continuous compact operators such that $T_{n} \rightarrow T$ (wot), where $T$ is $w^{*}-w$ continuous and compact. Then $T_{n} \rightarrow T$ weakly.

Proof. Let $A=\left\{\left(T_{n}\right), T\right\}$ and apply the previous lemma to this wotcompact set.

Next we apply $4.5-4.7$ to obtain an extension of Corollary 2 of [26]. 
THEOREM 4.8. If $H \subseteq L_{w^{*}}\left(E^{*}, F\right), H\left(x^{*}\right)$ is relatively weakly compact for each $x^{*} \in E^{*}, H^{*}\left(y^{*}\right)$ is relatively weakly compact for each $y^{*} \in F^{*}$, and $L_{w^{*}}\left(E^{*}, F\right)=K_{w^{*}}\left(E^{*}, F\right)$, then $H$ is relatively weakly compact.

Proof. Let $\left(h_{n}\right)$ be a sequence in $H$, and let $S=\left[h_{n}\left(E^{*}\right): n \geq 1\right]$. The compactness of each $h_{n}$ implies that $S$ is separable. Therefore $\left(B_{S^{*}}, w^{*}\right)$ is a compact metric space. Let $Y=\left(y_{n}^{*}\right)$ be a $w^{*}$-dense sequence in $S^{*}$. By hypotheses, $\left\{h_{n}^{*}\left(y_{i}^{*}\right): n \geq 1\right\}$ is relatively weakly compact for each $i$. By diagonalization, we may (and do) assume that $\left(h_{n_{i}}\right)$ is a subsequence of $\left(h_{n}\right)$ so that $\left(h_{n_{i}}^{*}\left(y_{k}^{*}\right)\right)_{i=1}^{\infty}$ is weakly convergent for each $k$. In fact, without loss of generality, we assume that $\left(h_{n}^{*}\left(y^{*}\right)\right)$ is weakly convergent for each $y^{*} \in Y$.

Now consider $\left(h_{n}\left(x^{*}\right)\right)$, where $x^{*} \in E^{*}$. This sequence must have a weakly convergent subsequence. Suppose that $z_{1}$ and $z_{2}$ are weak sequential cluster points of this sequence. Certainly $z_{1}, z_{2} \in S$. Suppose that $h_{k(n)}\left(x^{*}\right) \stackrel{w}{\rightarrow} z_{1}$, $h_{p(n)}\left(x^{*}\right) \stackrel{w}{\rightarrow} z_{2}$, and $y^{*} \in Y$. Now

$$
\begin{aligned}
\left\langle z_{1}, y^{*}\right\rangle & =\lim _{n}\left\langle h_{k(n)}\left(x^{*}\right), y^{*}\right\rangle=\lim _{n}\left\langle x^{*}, h_{k(n)}^{*}\left(y^{*}\right)\right\rangle \\
& =\lim _{n}\left\langle x^{*}, h_{p(n)}^{*}\left(y^{*}\right)\right\rangle=\lim _{n}\left\langle h_{p(n)}\left(x^{*}\right), y^{*}\right\rangle=\left\langle z_{2}, y^{*}\right\rangle,
\end{aligned}
$$

and $z_{1}=z_{2}$ since $Y$ is $w^{*}$-dense in $S^{*}$. Therefore $\left(h_{n}\left(x^{*}\right)\right)$ is $w$-convergent for all $x^{*} \in E^{*}$. Set

$$
h\left(x^{*}\right)=w-\lim h_{n}\left(x^{*}\right) \quad \text { for } x^{*} \in E^{*} .
$$

Next suppose that $y^{*} \in F^{*}$ and consider $h^{*}\left(y^{*}\right)$. Without loss of generality, suppose that $h_{n}^{*}\left(y^{*}\right) \stackrel{w}{\rightarrow} x \in E$, and let $x^{*} \in E^{*}$. Then $\left\langle h\left(x^{*}\right), y^{*}\right\rangle=$ $\lim _{n}\left\langle h_{n}\left(x^{*}\right), y^{*}\right\rangle=\lim _{n}\left\langle x^{*}, h_{n}^{*}\left(y^{*}\right)\right\rangle=x^{*}(x)$. Thus $h^{*}\left(y^{*}\right)=x$, and $h$ is $w^{*}-w$ continuous. Therefore $h$ is compact and $w^{*}-w$ continuous. Lemma 4.7 then shows that $h_{n} \stackrel{w}{\rightarrow} h$.

Corollary 4.9. If $E$ and $F$ are reflexive and $L(E, F)=K(E, F)$, then $K(E, F)$ is reflexive.

The assumption that $H$ is weakly precompact allows an interpretation of Theorem 4.8 for the space $L(X, Y)$.

Theorem 4.10. Suppose that $K(X, Y)=L(X, Y)$ and $H$ is a weakly precompact subset of $K(X, Y)$ so that

(i) $H(x)$ is relatively weakly compact for all $x \in X$,

(ii) $H^{*}\left(y^{*}\right)$ is relatively weakly compact for all $y^{*} \in Y^{*}$.

Then $H$ is relatively weakly compact.

Proof. Let $\left(T_{n}\right)$ be a sequence in $H$. Without loss of generality, suppose that $\left(T_{n}\right)$ is weakly Cauchy, and let $x \in X$. Since $\left(T_{n}(x)\right)$ has a weakly convergent subsequence and it is weakly Cauchy, it follows that it is weakly 
convergent. Similarly, $\left(T_{n}^{*}\left(y^{*}\right)\right)$ is weakly convergent for all $y^{*} \in Y^{*}$. Let $T \in L(X, Y)$ be such that $T_{n}(x) \stackrel{w}{\rightarrow} T(x)$ for all $x \in X$.

Since $T_{n}^{*}\left(y^{*}\right) \stackrel{w^{*}}{\rightarrow} T^{*}\left(y^{*}\right)$ and $\left\{T_{n}^{*}\left(y^{*}\right): n \geq 1\right\}$ is relatively weakly compact, one can show that $T_{n}^{*}\left(y^{*}\right) \stackrel{w}{\rightarrow} T^{*}\left(y^{*}\right)$, i.e. $T_{n} \stackrel{w^{\prime}}{\rightarrow} T$ in $K(X, Y)$. By Corollary 3 of Kalton [26], we obtain $T_{n} \rightarrow T$ weakly. Thus $H$ is relatively weakly compact.

Corollary 4.11. Suppose that $K_{w^{*}}\left(X^{*}, Y\right)=L_{w^{*}}\left(X^{*}, Y\right)$. If both $X$ and $Y$ have property $R D P^{*}$ (resp. the $(B D)$ property), then $K_{w^{*}}\left(X^{*}, Y\right)$ has property $R D P^{*}$ (resp. the (BD) property).

Proof. We present the proof of the result relative to the $\mathrm{RDP}^{*}$ property; the other case is similar. Let $H$ be a DP set in $K_{w^{*}}\left(X^{*}, Y\right)$. For each $x^{*} \in X^{*}$, $H\left(x^{*}\right)$ is a DP set in $Y$, thus relatively weakly compact. Similarly $H^{*}\left(y^{*}\right)$ is relatively weakly compact for each $y^{*} \in Y^{*}$. Apply Theorem 4.8.

Corollary 4.12. Suppose that $L(X, Y)=K(X, Y)$ and both $X^{*}$ and $Y$ have $R D P^{*}$ (resp. the $(B D)$ property). Then $K(X, Y)$ has $R D P^{*}$ (resp. the $(B D)$ property).

Corollary 4.13. Suppose that $L\left(X, Y^{*}\right)=K\left(X, Y^{*}\right)$ and both $X^{*}$ and $Y^{*}$ have $R D P^{*}$. Then $K\left(X, Y^{*}\right)$ has $R D P^{*}$ and $\ell_{1} \xi X \otimes_{\gamma} Y$.

Proof. $K\left(X, Y^{*}\right)$ has $\mathrm{RDP}^{*}$ by 4.12 . Note that $c_{0} \nrightarrow\left(X \otimes_{\gamma} Y\right)^{*}$ since $L\left(X, Y^{*}\right) \cong\left(X \otimes_{\gamma} Y\right)^{*}$ has $\operatorname{RDP}^{*}\left(c_{0}\right.$ does not have $\operatorname{RDP}^{*}$ since $\left(z_{n}\right)=$ $\left(\sum_{i=1}^{n} e_{i}\right)$ is a DP set which is not relatively weakly compact). By a result of Bessaga and Pełczyński, $\ell_{1} \stackrel{\xi}{\hookrightarrow} X \otimes_{\gamma} Y$.

The next three theorems continue to concentrate on conditions which ensure that spaces of operators have the $\mathrm{RDP}^{*}$ or the (BD) property.

TheOREM 4.14. Suppose that $L\left(X, Y^{*}\right)=K\left(X, Y^{*}\right)$ and $Y^{*}$ has $R D P^{*}$. The following statements are equivalent:

(i) $X^{*}$ has $R D P^{*}$ and $\ell_{1} \hookrightarrow X$ or $\ell_{1} \hookrightarrow Y$.

(ii) $K\left(X, Y^{*}\right)$ has $R D P^{*}$.

Proof. Suppose first that $X^{*}$ has $\mathrm{RDP}^{*}$ and $\ell_{1} \nrightarrow X$ or $\ell_{1} \nrightarrow Y$. By 4.13, $K\left(X, Y^{*}\right)$ has $\mathrm{RDP}^{*}$.

Now suppose that $K\left(X, Y^{*}\right)$ has $\mathrm{RDP}^{*}$. Then $K\left(Y, X^{*}\right)$ has $\mathrm{RDP}^{*}$ since $K\left(Y, X^{*}\right) \cong K\left(X, Y^{*}\right)$. Thus $X^{*}$ has $\mathrm{RDP}^{*}$ since the property $\mathrm{RDP}^{*}$ is inherited by subspaces. We will show that $\ell_{1} \nrightarrow X$ or $\ell_{1} \nrightarrow Y$. Suppose that $\ell_{1} \hookrightarrow X$ and $\ell_{1} \hookrightarrow Y$. Hence $L^{1}$ and thus $\ell_{2}$ embeds in $X^{*}$ and similarly $\ell_{2}$ embeds in $Y^{*}$. Hence $\ell_{2} \otimes_{\lambda} \ell_{2}$ is isomorphic to a subspace of $L\left(X, Y^{*}\right)=$ $K\left(X, Y^{*}\right)$ and thus $\ell_{2} \otimes_{\lambda} \ell_{2}$ has $\mathrm{RDP}^{*}$. By Lemma 3.2 of [23] (as well as a result of Emmanuele [18]), $c_{0} \hookrightarrow \ell_{2} \otimes_{\lambda} \ell_{2}$, and we have a contradiction since $c_{0}$ fails $\mathrm{RDP}^{*}$. 
THEOREM 4.15 .

(i) Suppose that $X$ has the Schur property and $Y$ has $R D P^{*}$. Then $L_{w^{*}}\left(X^{*}, Y\right)=K_{w^{*}}\left(X^{*}, Y\right)$ has $R D P^{*}$ and $c_{0} \nrightarrow K_{w^{*}}\left(X^{*}, Y\right)$.

(ii) Suppose that $X$ has $R D P^{*}$ and $Y$ has the Schur property. Then $L_{w^{*}}\left(X^{*}, Y\right)=K_{w^{*}}\left(X^{*}, Y\right)$ has $R D P^{*}$ and $c_{0} \longleftrightarrow K_{w^{*}}\left(X^{*}, Y\right)$.

(iii) Suppose that $X$ has the Schur property and $Y$ has the $(B D)$ property. Then $L_{w^{*}}\left(X^{*}, Y\right)=K_{w^{*}}\left(X^{*}, Y\right)$ has the $(B D)$ property.

(iv) Suppose that $X$ has the $(B D)$ property and $Y$ has the Schur property. Then $L_{w^{*}}\left(X^{*}, Y\right)=K_{w^{*}}\left(X^{*}, Y\right)$ has the $(B D)$ property.

Proof. We present the proof of (i); the other cases are similar. Let $T \in$ $L_{w^{*}}\left(X^{*}, Y\right)$. Then $T$ is weakly compact since $T^{*}$ is $w^{*}-w$ continuous. Hence $T^{*}$ is compact since $X$ is a Schur space. An application of 4.11 shows that $K_{w^{*}}\left(X^{*}, Y\right)$ has $\mathrm{RDP}^{*}$.

THEOREM 4.16.

(i) Suppose that $X^{*}$ is a Schur space and $Y$ has $R D P^{*}$. Then $K(X, Y)=$ $L(X, Y)$ has $R D P^{*}$ and $c_{0} \nrightarrow K(X, Y)$. Moreover, if $Y=Z^{*}$, then $\ell_{1} \stackrel{q}{\varphi}$ $X \otimes_{\gamma} Z$.

(ii) Suppose that $X^{*}$ has $R D P^{*}$ and $Y$ is a Schur space. Then $K(X, Y)=$ $L(X, Y)$ has $R D P^{*}$ and $c_{0} \hookrightarrow K(X, Y)$. Moreover, if $Y=Z^{*}$, then $\ell_{1} \&$ $X \otimes_{\gamma} Z$.

(iii) Suppose that $X^{*}$ is a Schur space and $Y$ has the $(B D)$ property. Then $K(X, Y)=L(X, Y)$ has the $(B D)$ property.

(iv) Suppose that $X^{*}$ has the $(B D)$ property and $Y$ is a Schur space. Then $K(X, Y)=L(X, Y)$ has the $(B D)$ property.

Proof. We present the proofs for (i) and (ii) only.

Let $T \in L(X, Y)$. Since $X^{*}$ is a Schur space, $T^{*}$ is completely continuous, and $T\left(B_{X}\right)$ is a DP set in $Y$, thus relatively weakly compact. Then $T$ and hence $T^{*}$ is weakly compact, and therefore compact because $X^{*}$ has the Schur property. By 4.12, $K(X, Y)=L(X, Y)$ has RDP* and $c_{0} \nrightarrow K(X, Y)$.

Suppose now that $Y=Z^{*}$. Then $c_{0} \hookrightarrow K\left(X, Z^{*}\right) \cong\left(X \otimes_{\gamma} Z\right)^{*}$ and thus $\ell_{1} \stackrel{\&}{\Psi} X \otimes_{\gamma} Z$. Another application of 4.12 gives (ii).

REMARK. Theorems 4.15 and 4.16 provide examples of spaces of operators which contain $\ell_{1}$ and have the $(\mathrm{BD})$ property.

Corollary 4.17. Suppose that $X$ has $R D P^{*}$ (resp. the (BD) property). Then the space $l^{1}[X]$ of all unconditionally convergent series in $X$ with the norm

has $R D P^{*}$ (resp. the $(B D)$ property).

$$
\left\|\left(x_{n}\right)\right\|=\sup \left\{\sum\left|x^{*}\left(x_{n}\right)\right|: x^{*} \in B_{X^{*}}\right\}
$$


Proof. It is known that $\ell_{1}[X]$ is isometrically isomorphic to $K\left(c_{0}, X\right)$ [20]. Since $X$ has $\mathrm{RDP}^{*}$ and $c_{0}^{*}=\ell_{1}$ has the Schur property, Theorem 4.16 gives the conclusion.

The fact that the continuous linear image of a Dunford-Pettis (resp. limited) set is Dunford-Pettis (resp. limited) produces the following result for quotient spaces.

Theorem 4.18. If $X^{*}$ has $R D P^{*}$ (resp. the $(B D)$ property) and $Z$ is a quotient of $X$, then $Z^{*}$ has $R D P^{*}$ (resp. the $(B D)$ property).

Proof. Let $Q: X \rightarrow Z$ be a quotient map. Then $Q^{*}: Z^{*} \rightarrow X^{*}$ is an isomorphism. If $K$ is a DP subset of $Z^{*}$, then $Q^{*}(K)$ is a DP subset of $X^{*}$. Thus $Q^{*}(K)$ and $K$ must be relatively weakly compact.

Corollary 4.19. Suppose that $L\left(X^{*}, Y^{*}\right)=K\left(X^{*}, Y^{*}\right)$ and both $X^{* *}$ and $Y^{*}$ have $R D P^{*}$. Then the dual of the space $N_{1}(X, Y)$ of all nuclear operators has $R D P^{*}$ and hence $\ell_{1} \stackrel{\leftrightarrow}{\leftrightarrow} N_{1}(X, Y)$.

Proof. It is known that $N_{1}(X, Y)$ is a quotient of $X^{*} \otimes_{\gamma} Y$ ([16]). By Corollary 4.13, $\left(X^{*} \otimes_{\gamma} Y\right)^{*} \cong L\left(X^{*}, Y^{*}\right)$ has RDP*. An application of 4.18 gives that $\left(N_{1}(X, Y)\right)^{*}$ has $\mathrm{RDP}^{*}$. Thus $c_{0} \hookrightarrow\left(N_{1}(X, Y)\right)^{*}$ and $\ell_{1} \stackrel{4}{\varphi}$ $N_{1}(X, Y)$.

Feder [22] and Emmanuele [19] asked if there exist spaces $X$ and $Y$ so that $c_{0} \nrightarrow K(X, Y)$ yet $L(X, Y) \neq K(X, Y)$. Emmanuele gave a positive solution to this question in the concluding "added in proof" remark in [19] using a fundamental space created by Bourgain and Delbaen [5]. Results of this note highlight additional properties of the Bourgain-Delbaen spaces as well as operators defined on these spaces. We denote the two spaces by $\mathcal{X}$ and $\mathcal{Y}$. Both $\mathcal{X}$ and $\mathcal{Y}$ are infinite-dimensional spaces with the DunfordPettis property. The space $\mathcal{X}$ is a separable $\mathcal{L}_{\infty}$-space which is a Schur space, and both $\mathcal{X}$ and $\mathcal{X}^{*}$ are weakly sequentially complete. Since $\mathcal{X}$ is a Schur space and $\mathcal{X}^{*}$ is weakly sequentially complete, $\mathcal{X}$ contains a copy of $\left(e_{n}^{*}\right)$ no subsequence of which can be complemented in $\mathcal{X}$. Thus by Theorem 3.9, $\left(e_{n}^{*}\right)$ embeds as a $V^{*}$-sequence in $\mathcal{X}$.

The space $\mathcal{Y}$ is a separable $\mathcal{L}_{\infty}$-space which is somewhat reflexive. Further, $\mathcal{Y}^{*}$ is isomorphic to $\ell_{1}$. Consequently, there is a sequence $\left(y_{n}^{*}\right)$ in $\mathcal{Y}^{*}$ so that $\left(y_{n}^{*}\right) \sim\left(e_{n}^{*}\right),\left(y_{n}^{*}\right)$ is a $V$-sequence in $\mathcal{Y}^{*}$, and $\left[y_{n}^{*}: n \in A\right]$ is complemented in $\mathcal{Y}^{*}$ for each non-empty $A \subseteq \mathbb{N}$.

If the Banach spaces $Z$ and $Z^{*}$ are weakly sequentially complete and

$$
L_{w^{*}}\left(Z^{* *}, Z\right)=K_{w^{*}}\left(Z^{* *}, Z\right)
$$

then $K_{w^{*}}\left(Z^{* *}, Z\right)$ is weakly sequentially complete. Since

$$
K(Z, Z) \cong K_{w^{*}}\left(Z^{* *}, Z\right),
$$


$K(Z, Z)$ is weakly sequentially complete. If $Z$ is infinite-dimensional, then certainly $L(Z, Z) \neq K(Z, Z)$ and $c_{0} \nrightarrow K(Z, Z)$. Setting $Z=\mathcal{X}$, one obtains Emmanuele's solution [19].

Alternatively, suppose that $X^{*}$ is weakly sequentially complete, $Y$ is a Schur space, and $L(X, Y) \neq K(X, Y)$. Then $K(X, Y) \cong K_{w^{*}}\left(X^{* *}, Y\right)=$ $L_{w^{*}}\left(X^{* *}, Y\right)$, and 4.4 implies that $L_{w^{*}}\left(X^{* *}, Y\right)$ is weakly sequentially complete. Thus $c_{0} \nrightarrow K(X, Y)$.

If $\ell_{1} \hookrightarrow E$ and $F$ is arbitrary, then every operator $T: E \rightarrow F$ is almost weakly compact. If $F$ is weakly sequentially complete, then the operator $T$ is weakly compact. If $F$ is Schur, then $T$ is compact. Consequently, if $F$ is Schur, then $L(\mathcal{Y}, F)=K(\mathcal{Y}, F)$. Specifically, note that $L(\mathcal{Y}, \mathcal{X})=K(\mathcal{Y}, \mathcal{X})$. Further, an immediate application of the Lust-Ryan theorem stated earlier shows that $L(\mathcal{Y}, \mathcal{X})$ is a Schur space and thus is weakly sequentially complete. Consequently,

$$
c_{0} \nrightarrow L(\mathcal{Y}, \mathcal{X})
$$

Moreover, another application of Theorem 4.4 shows that

$$
K_{w^{*}}\left(\mathcal{X}^{* *}, L(\mathcal{Y}, \mathcal{X})\right)=L_{w^{*}}\left(\mathcal{X}^{* *}, L(\mathcal{Y}, \mathcal{X})\right)
$$

is weakly sequentially complete. Therefore

$$
c_{0} \hookrightarrow K(\mathcal{X}, L(\mathcal{Y}, \mathcal{X})) \cong K_{w^{*}}\left(\mathcal{X}^{* *}, L(\mathcal{Y}, \mathcal{X})\right)
$$

Since $\mathcal{X}$ embeds isometrically in $L(\mathcal{Y}, \mathcal{X})$,

$$
L(\mathcal{X}, L(\mathcal{Y}, \mathcal{X})) \neq K(\mathcal{X}, L(\mathcal{Y}, \mathcal{X}))
$$

and we have another example of a solution to the Feder-Emmanuele problem discussed previously.

The proof of Theorem 4.14 made use of the fact that $c_{0} \hookrightarrow \ell_{2} \otimes_{\lambda} \ell_{2}$ and Corollary 1.3.6 of [35] showed that if $c_{0} \hookrightarrow X$, then $c_{0}$ is complemented in $X \otimes_{\lambda} X$. Certainly $c_{0} \hookrightarrow \mathcal{X}$, and the Lust-Ryan theorem (or Theorem 4.4) easily shows that the least crossnorm tensor product completion of any Schur space with itself does not contain $c_{0}$. Specifically, $\mathcal{X} \otimes_{\lambda} \mathcal{X} \hookrightarrow L_{w^{*}}\left(\mathcal{X}^{*}, \mathcal{X}\right)$, and Theorem 4.4 shows that this space of operators is weakly sequentially complete, while the Lust-Ryan result shows even more. This space of operators is a Schur space; thus $\mathcal{X} \otimes_{\lambda} \mathcal{X}$ is a Schur space.

\section{References}

[1] K. Andrews, Dunford-Pettis sets in the space of Bochner integrable functions, Math. Ann. 241 (1979), 35-41.

[2] E. M. Bator, Remarks on completely continuous operators, Bull. Polish Acad. Sci. Math. 37 (1989), 409-413.

[3] E. Bator, P. Lewis, and J. Ochoa, Evaluation maps, restriction maps, and compactness, Colloq. Math. 78 (1998), 1-17. 
[4] F. Bombal, On $V^{*}$ sets and Petczyński's property $V^{*}$, Glasgow Math. J. 32 (1990), 109-120.

[5] J. Bourgain and F. Delbaen, A class of special $\mathcal{L}_{\infty}$ spaces, Acta Math. 145 (1981), $155-176$.

[6] J. Bourgain and J. Diestel, Limited operators and strict cosingularity, Math. Nachr. 119 (1984), 55-58

[7] J. Brooks and P. Lewis, Linear operators and vector measures, Trans. Amer. Math. Soc. 192 (1974), 139-162.

[8] W. J. Davis, D. W. Dean, and B.-L. Lin, Bibasic sequences and norming basic sequences, Trans. Amer. Math. Soc. 176 (1974), 89-102.

[9] J. Diestel, A survey of results related to the Dunford-Pettis property, in: Contemp. Math. 2, Amer. Math. Soc., 1980, 15-60.

[10] - Sequences and Series in Banach Spaces, Grad. Texts in Math. 92, Springer, Berlin, 1984.

[11] J. Diestel and J. J. Uhl, Jr., Vector Measures, Math. Surveys 15, Amer. Math. Soc., 1977.

[12] I. Dobrakov, On representation of linear operators on $C_{0}(T, X)$, Czechoslovak Math. J. 21 (1971), 13-30.

[13] L. Drewnowski and G. Emmanuele, On Banach spaces with the Gelfand-Phillips property, II, Rend. Circ. Mat. Palermo 38 (1989), 377-391.

[14] G. Emmanuele, A dual characterization of Banach spaces not containing $\ell^{1}$, Bull. Polish Acad. Sci. Math. 34 (1986), 155-160.

[15] -, On Banach spaces with the property $\left(V^{*}\right)$ of Petczyński, Ann. Mat. Pura Appl. 152 (1988), 171-181.

[16] - The reciprocal Dunford-Pettis property and projective tensor products, Math. Proc. Cambridge Philos. Soc. 109 (1991), 161-166.

[17] - On Banach spaces containing complemented copies of $c_{0}$, Extracta Math. 3 (1988), 98-100.

[18] - On the containment of $c_{0}$ in spaces of compact operators, Bull. Sci. Math. 115 (1991), 177-184.

[19] - A remark on the containment of $c_{0}$ in spaces of compact operators, Math. Proc. Cambridge Philos. Soc. 111 (1992), 331-335.

[20] - Banach spaces in which Dunford-Pettis sets are relatively compact, Arch. Math. (Basel) 58 (1992), 477-485.

[21] - About the position of $K_{w^{*}}\left(E^{*}, F\right)$ inside $L_{w^{*}}\left(E^{*}, F\right)$, Atti Sem. Mat. Fis. Univ. Modena 42 (1994), 123-133.

[22] M. Feder, On the nonexistence of a projection onto the space of compact operators, Canad. Math. Bull. 25 (1982), 78-81.

[23] I. Ghenciu and P. Lewis, Strong Dunford-Pettis sets and spaces of operators, Monatsh. Math. 144 (2005), 275-284.

[24] A. Grothendieck, Critères de compacité dans les espaces fonctionnels généraux, Amer. J. Math. 74 (1952), 168-186.

[25] R. Haydon, A non-reflexive Grothendieck space that does not contain $\ell_{\infty}$, Israel J. Math. 40 (1981), 65-73.

[26] N. Kalton, Spaces of compact operators Math. Ann. 208 (1974), 267-278.

[27] P. Lewis, Spaces of operators and $c_{0}$, Studia Math. 145 (2001), 213-218.

[28] —, Dunford-Pettis sets, Proc. Amer. Math. Soc. 129 (2001), 3297-3302.

[29] J. Lindenstrauss and L. Tzafriri, Classical Banach Spaces, I, Ergeb. Math. Grenzgeb. 92, Springer, Berlin, 1977. 
[30] F. Lust, Produits tensoriels injectifs d'espaces de Sidon, Colloq. Math. 32 (1975), 286-289.

[31] A. Pełczyński, Banach spaces on which every unconditionally converging operator is weakly compact, Bull. Acad. Polon. Sci. Sér. Sci. Math. Astronom. Phys. 10 (1962), 641-648.

[32] A. Pietsch, Absolut p-summierende Abbildungen in normierten Räumen, Studia Math. 28 (1966/1967), 333-353.

[33] H. Rosenthal, Pointwise compact subsets of the first Baire class, Amer. J. Math. 99 (1977), 362-377.

[34] R. Ryan, The Dunford-Pettis property and projective tensor products, Bull. Polish Acad. Sci. Math. 35 (1987), 785-792.

[35] T. Schlumprecht, Limited sets in Banach spaces, Dissertation, Munich, 1987.

[36] I. Singer, Bases in Banach Spaces, II, Springer, 1981.

[37] C. Swartz, Unconditionally converging operators on the space of continuous functions, Rev. Roumaine Math. Pures Appl. 17 (1973), 123-131.

Ioana Ghenciu

Department of Mathematics

University of Wisconsin at River Falls

River Falls, WI 54022, U.S.A.

E-mail: ioana.ghenciu@uwrf.edu
Paul Lewis

Department of Mathematics University of North Texas

Box 311430

Denton, TX 76203-1430, U.S.A. E-mail: lewis@unt.edu

Received June 9, 2006;

received in final form November 22, 2006 